\title{
Kernos
}

Revue internationale et pluridisciplinaire de religion grecque antique

3 | 1990

Varia

\section{Les oracles delphiques relatifs aux pestilences et Thucydide}

\section{Paul Demont}

\section{OpenEdition \\ Journals}

Édition électronique

URL : http://journals.openedition.org/kernos/982

DOI : $10.4000 /$ kernos.982

ISSN : 2034-7871

\section{Éditeur}

Centre international d'étude de la religion grecque antique

\section{Édition imprimée}

Date de publication : 1 janvier 1990

ISSN : 0776-3824

Référence électronique

Paul Demont, "Les oracles delphiques relatifs aux pestilences et Thucydide», Kernos [En ligne],

3 | 1990, mis en ligne le 19 avril 2011, consulté le 30 avril 2019. URL : http://journals.openedition.org/ kernos/982 ; DOI : 10.4000/kernos.982 


\section{LES ORACLES DELPHIQUES RELATIFS AUX PESTILENCES ET THUCYDIDE}

Le Colloque qui nous réunit aujourd'hui est l'occasion d'aborder par un nouveau biais un sujet - la description faite par Thucydide de la pestilence athénienne de 430-426 avant J.-C. - que j'ai jusqu'ici étudié dans une perspective différente ${ }^{1}$, plus à partir de la Collection hippocratique qu'en rapport avec la conception religieuse des pestilences, qui ne me semblait guère faire problème. Or voici que notre Colloque m'incite à approfondir ce dernier aspect.

La civilisation grecque n'est certes pas la seule à avoir interprété les "brusques épidémies mortelles» ${ }^{2}$ qui surviennent dans une population donnée "comme des signes d'intervention divine" : c'était aussi le cas, entre autres exemples, et pour rester dans la même période chronologique, des «rédacteurs de l'Ancien Testament, lorsqu'ils donnèrent, entre 1000 et 500 avant Jésus-Christ, sa forme actuelle au texte».

Je limiterai mon étude aux traditions concernant les oracles delphiques $^{3}$ et à ce qu'elles apportent à mon avis à la lecture du récit de

1 Notes sur le récit de la pestilence athénienne chez Thucydide et sur ses rapports avec la médecine grecque de l'époque classique, in Actes du TVe Colloque international hippocratique (Lausanne), éd. F. LAsserRe et Ph. MUDRY, Genève, Droz, 1983, p. 341-354; Hérodote et les pestilences (Note sur Hdt. VI, 27; VII, 171 et VIII, 115-117), in RPh, 62 (1988), p. 7-13.

2 Ceci est une très bonne définition de ce qu'est un loimos. Cette citation et la suivante sont empruntées au livre classique de William H. MacNeILL, Le temps de la peste, Essai sur les épidémies dans l'Histoire, trad. Paris, Hachette, 1978 (= Anchor Press, 1976), p. 77, qui donne beaucoup d'autres références. Pour l'Ancien Testament, signalons seulement Ex. IX (cinquième plaie d'Égypte) repris par ex. dans ÉzéCHIEL (TrGF t. I, 128 Ex. 140); et pour le Nouveau Testament, MatTHIEU, XXIV, 7 (mais voir note 8). Ajoutons, pour le monde grec, H. F. J. Hortsmanshof, Pestilenties in de Griekse wereld, in Lampas, 17 (1984), p. 433-452 (je n'ai pu consulter la thèse publiée récemment par l'auteur sur le sujet : De Pÿlen van de Pest. Pestilenties in de Griekse Wereld (800-400 v.c.), Amsterdam, 1989, 299 p.) et pour le monde latin, J.-M. ANDRE, La notion de pestilentia à Rome : du tabou religieux à l'interprétation préscientifique, in Latomus, 39 (1980), p. 3-16.

3 H.W. PARke, D.E.W. Wormell, The Delphic Oracle II : The Oracular Responses, Oxford, Blackwell, 1956 (cité ici : PWo); J. FonTENRose, The Delphic Oracle, Univ. of California Press, 1978, 476 p. (cité ici : Fontenrose). Il ne faut pas oublier que ces oracles ne représentent cependant qu'une infime 
la pestilence athénienne chez Thucydide : pour comprendre ce récit, de même qu'il est nécessaire de comprendre ce que pouvait être un loimos pour les esprits éclairés, rationalistes, les médecins du temps de Thucydide, de la même façon, il est peut-être utile d'étudier les croyances relatives aux pestilences; l'originalité de l'historien n'en apparaîtra que mieux.

Ma première observation concerne le rapport entre loimos «pestilence» et limos «famine». Le répertoire thématique de J. Fontenrose propose, sous l'entrée "plague, famine, drought, catastrophe», le chiffre impressionnant de soixante-dix-neuf attestations d'oracles delphiques, pour la plupart peu ou pas historiques ${ }^{4}$. Les termes de l'entrée ne distinguent que deux des fléaux qui sont comparables aux pestilences : la famine (limos) et la sécheresse, qui détruisent la vie des plantes et des hommes; parmi les autres catastrophes, plusieurs pourraient être groupées, qui sont liées à la stérilité ou à la mauvaise reproduction des animaux et des hommes 5 . Dans les oracles delphiques,

partie des activités mantiques et des collections d'oracles rédigées (cf. FONTENROSE, p. 145-165, avec bibl.), dont beaucoup concernaient aussi les pestilences. Concernant Apollon, voir ses épiclèses de Loimios (à Lindos), Alexikakos (à Athènes, cf. PAUS., I, 3, 4), ainsi que, sans rapport explicite avec les pestilences : akestôr, akesôdynos, euphrôn, iètèr nosôn, nousolytès. Je ne fais que rarement intervenir le caractère historique ou légendaire des oracles : l'essentiel pour mon propos est l'existence de traditions anciennes bien attestées.

4 P. 442. Voici parmi ces références celles qui concernent spécifiquement les pestilences : Q 1 (PWo 485), 2 (486), 3 (487), 4 (488), 54 (223), 65 (13), 72 (237), 79 (p. 72 ad 164), 82 (27), 84 (552), 94 (401), 107 (58), 126 (75), 133 (572), 164 (113), 174 (114), 189 (125), 190 (158), 200 (179), 229 (353); L 19 (PWo 150, 479), 35 (199), 42 (207), 44 (209), 45 (210), 64 (291-292), 72 (305), 88 (376), 92 (386), 98 (405), $126(530), 133(542-543), 134$ (556), 140 (570), 145 (155), 153 (563), 157 (332), 159 (545-546), 169 (540), 173 (551). Voir aussi, à propos de Synésios de Cyrène, Lettres, XLI, 53-56 et XLII, 71, D. RoQUES, Synésios de Cyrène et la Cyrénaïque du Bas-Empire, Paris, C.N.R.S., 1988, p. 39.

5 Marie DELCOURT a attiré l'attention sur ce dernier aspect dans son livre sur Stérilités mystérieuses et naissances maléfiques dans l'Antiquité classique, Paris-Liège, 1938, mais elle refuse, à tort, semble-t-il, que le loimos soit une maladie (elle omet plusieurs textes précis à cet égard, en particulier THUC., I, 23, 3 , et les textes hippocratiques, et propose de plusieurs autres, en particulier de l'Edipe-Roi de Sophocle, des interprétations qui paraissent forcées). Voir aussi J.-P. VERnANT, Mythe et Tragédie en Grèce ancienne, Paris, 1972, p. 117 sq. et nos Notes... sur Thucydide (citées n. 1), p. 343 et n. 9. Quelques oracles sur les malformations, la stérilité humaine et les imperfections de la génération (sans mention de loimos) : Q 113 (PWo 64, HDT., I, 167, 2), Q 132 (PWo 83, HDT., VI, 
on observe d'abord que l'association du loimos et du limos se rencontre plusieurs fois dans des termes qui sont proches des formules d'Hésiode et d'Hérodote ${ }^{6}$. J'ai tenté ailleurs de montrer qu'Hérodote et Thucydide sont marqués par cette association, même s'ils s'efforcent, chacun une fois, et chacun différemment, d'en rendre compte de façon rationnellement admissible, "Thucydide en donnant une cause commune, la sécheresse, aux deux fléaux, Hérodote, en suggérant une explication par le régime sauvage auquel la famine contraint» 7 . Je n'ajoute ici qu'un point de détail. On peut observer qu'une hésitation entre le loimos et le limos se rencontre à plusieurs reprises dans les traditions concernant certains oracles delphiques, et dans la tradition manuscrite de certains textes ${ }^{8}$. Selon les versions, c'est une famine ou une pestilence qui frappe

VI, 139, 2), Q 161 (PWo 108, HDT., IX, 93, 4), Q 209 (PWo 554, PAUs., III, 18, 1), Q 232 (PWo 333), L 91 (PWo 385) Fontenrose. Sur HDT., I, 167, cf. dernièrement J.-P. THUILLIER, Les conséquences de la bataille d'Alalia (Hdt. I, 167) : oracle delphique et divination étrusque, in Caesarodunum (1985), suppl. 52 ( $L a$ divination dans le monde étrusco-italien), p. 23-31; mais Hérodote ne parle pas dans ce cas de loimos; d'autre part, l'interprétation de J.-P. THUILLIER, fort vraisemblable dans l'ensemble, est inexacte sur un point : il y a des exemples de jeux prescrits par la Pythie pour la Grèce, pour le monde grec, pour expier des fautes et guérir des épidémies : voir certaines légendes sur la refondation des Jeux Olympiques citées infra et THUC., III, 104.

6 Q 3 (PWo 487), Q 82 PWo 27), L 45 (PWo 210), L 72 (PWo 305), L 134 (PWo 556) et L 140 (PWo 570), à comparer à HÉsIODE, Trav., 243 et HDT., VII, 171 (cf. Q 145 Fontenrose = PWo 93). Les textes latins associent de même souvent fames et pestilentia (cf. J.-M. ANDRÉ, op. cit., p. 4-5), ce qui prouve qu'il ne s'agit pas seulement, dans le cas du grec, d'une confusion entre deux mots proches (voire homophones en raison de l'iotacisme).

7 Voir THuc., I, 23, 3, et HDT., VIII, 115-117, ainsi que les deux travaux cités n. 1.

8 Voici quelques exemples; dans certains cas, il peut s'agir de confusions dues à l'iotacisme et à l'homophonie; mais le plus souvent, il y a plutôt équivalence fonctionnelle entre les deux fléaux : Q 164 Fontenrose (PWo 113) : loimos ou limos selon les scholiastes pour le fléau ayant nécessité le retour des os de Thésée à Athènes en 476-475 av. J.-C.; Callimaque, H. Artémis, v. 125 (le poète imite Hésiode, chez qui on a à la fois limos et loimos, mais n'a, lui, qu'un seul mot : certains manuscrits donnent limos, d'autres loimos); MATTHIEU, XXIV, 7 (il s'agit des prophéties sur la fin des temps : selon la famille de manuscrits, on lit limoi kai seismoi ou limoi kai loimoi kai seismoi); APOLlODORE : la même calamité, appelée à II, 7, 4, loimos, est à III, 9, 1, akarpou tès gès menousès (cité par M. DELCOURT, op. cit., p. 26, qui en tire argument pour soutenir que loimos signifie akarpia); comparer Exode IX au texte du poète tragique ÉzÉCHIEL cité n. 3 (dans ce cas, on a comme une contamination du texte biblique par les traditions gréco-latines concernant les pestilences humaines); voir aussi LIBANIOs, Vie, 284-285, et les scholies à ARISTOPHANE, Cav., 728. 
un pays. Or chacun connaît le texte dans lequel Thucydide, à la fin de sa description de la pestilence, cite un vieil oracle en hexamètre que les plus âgés des Athéniens se rappellent avoir appris :

Dans cette épreuve, les gens rappelaient naturellement des souvenirs, évoquant le vers qu'au dire des plus âgés on récitait autrefois : On verra arriver la guerre dorienne et avec elle la pestilence (loimos). En fait, il y eut désaccord : le mot figurant autrefois dans le vers n'aurait pas été pestilence (loimos) mais disette (limos); pourtant l'avis qui naturellement prévalut fut en l'occurrence que le mot était pestilence (loimos). Les gens réglaient en effet leurs souvenirs sur ce qui leur arrivait; et si, j'imagine, il se présente jamais une autre guerre dorienne après celle-ci et qu'il se trouve y avoir disette (limos), c'est naturellement sous cette forme que l'on fera la citation 9 .

Les trois «naturellement» (eikos) soulignent la fragilité de la mémoire humaine et la façon dont elle adapte les prédictions aux faits. Thucydide manifeste encore ici son attention à un aspect de la représentation traditionnelle des pestilences, le rapport, voire l'hésitation, entre famines et pestilences : d'un côté, comme je l'ai suggéré par ailleurs, il tente d'en rendre compte de façon rationnelle au livre I chap. 23,3 , en donnant une cause commune aux deux fléaux, de l'autre, ici, il prend ses distances à l'égard de certaines confusions fréquentes dès son temps entre les deux et les commente dans la perspective de son anthropologie.

Ma seconde observation porte sur l'une des causes du loimos pour lequel on consulte l'oracle de Delphes, à savoir un meurtre impie et le non-respect des rites de sépulture ${ }^{10}$. Plusieurs exemples illustrent ce

9 II, 54, 2-3, trad. J. de Romilly, modifiée sur un point (C.U.F., 1962). A.W. GOMME dans son Historical Commentary on Thucydides, vol. II, Oxford, Clarendon Press, 1962, p. 161, accorde une certaine valeur à un raisonnement selon lequel ce texte doit être antérieur à 405-404 (année de famine terrible à Athènes); mais la famine de 405-404 n'a rien à voir avec une malédiction pesant sur les récoltes, seule visée dans l'oracle.

10 Le cas le plus célèbre est la pestilence thébaine après le meutre de Laïos par Edipe; mais la question de la sépulture n'y joue aucun rôle, du moins dans la version de Sophocle. Le Dr Grmek me fait observer que la croyance dans le caractère malsain et morbifique des cadavres sans sépulture, qui n'est fondée sur aucune réalité, perdure encore jusqu'à nos jours. 
lien. C'est, selon l'une des versions transmises par Diogène Laërce, ce qui arriva à Athènes après le meurtre de Cylon et ce qui conduisit à faire appel à Épiménide, probablement en 596 avant J.-C. ${ }^{11}$; au milieu du sixième siècle ${ }^{12}$, les Delphiens furent atteints par une pestilence à la suite du meurtre d'Ésope à Delphes; vers la même époque, le tyran Pythagoras d'Éphèse tua des adversaires politiques réfugiés dans des temples et refusa leur sépulture; une fille, qu'il avait épargnée, se pendit dans le sanctuaire pour ne pas mourir de faim : Éphèse fut ravagée par la pestilence et la famine ${ }^{13}$; selon Aristodème, le meurtre de Pausanias (Thuc., I, 134, 4) attira aussi sur Sparte un loimos ${ }^{14}$. La Piérie fut ravagée par la pestilence, selon l'historien Conon, après le meurtre d'Orphée ${ }^{15}$, tout comme Corinthe après le meurtre des enfants de Médée ${ }^{16}$, selon un scholiaste d'Euripide; pour effacer la souillure, il fallut dans chaque cas un rituel de sépulture. Un cas célèbre est celui du loimos et du limos qui frappèrent Athènes après la mort d'Androgée, fils de Minos; Athènes dut payer tribut en offrant chaque année à Minos sept jeunes garçons et sept jeunes filles ${ }^{17}$; ce meurtre explique aussi le rituel de l'expulsion du pharmakos aux Thargélies d'Athènes, selon certains $^{18}$, ou bien encore le culte de Zeus Panellenios à Égine. Dans certains cas, la pestilence naît parce que des ossements ne sont pas enterrés dans le sol qui devrait les avoir reçus, ainsi pour les ossements de Pélops à Élée ${ }^{19}$, de Thésée à Athènes selon certains ${ }^{20}$ et ceux d'Hésiode à Orchomène ${ }^{21}$. Naturellement, d'autres légendes dévelop-

11 Q 65 Fontenrose (PWo 13); en 500 avant J.-C. selon PlaTON (Lois, 642d).

12 Q 107 Fontenrose (PWo 58) : HÉRODOTE y fait allusion (II, 134, 4).

13 Q 82 et voir p. 76-77 Fontenrose (PWo 27); le mot loimos ne figure pas dans la source, mais seulement $\delta \eta \mu$ oría vóøos.

14 Q 174 (PWo 114); cf. FGrH 104 F 1, 8.

15 L 88 Fontenrose (PWo 376); cf. FGrH 26 F 1, 45, 5 et 131 F 2 (cf. PAUs., X, 7, 2; $\mathrm{XXX}, 6,8)$.

16 L 35 Fontenrose (PWo 199); cf. les scholies à Eur., Méd., 273.

17 L 45-46 Fontenrose (PWo 210-211).

18 Voir les témoignages et leur analyse dans J.-P. VERNANT, op. cit., p. 117-118.

19 L 153 Fontenrose (PWo 563); cf. PAUS., V, 13, 5.

20 Q 164 Fontenrose (PWo 113) : l'affaire se situe peut-être au moment de la prise de Skyros par Cimon (476/5 avant J.-C.) ou bien est uniquement légendaire (cf. p. 73-74 Fontenrose); voir note 9.

21 L 42 Fontenrose (PWo 207); cf. PAUS., IX, 38, 3. 
pent ce thème, qui repose, comme on sait, sur la croyance en la contagion de la souillure ${ }^{22}$, sans qu'un loimos intervienne nécessairement.

Revenons maintenant à Thucydide. Dans sa description de la pestilence athénienne, il y a quelques lignes particulièrement insistantes et spectaculaires sur l'accumulation des cadavres dans Athènes :

Des corps gisaient, au moment de mourir, les uns sur les autres; il y en avait qui se roulaient par terre, à demi morts, sur les chemins et vers toutes les fontaines, mus par le désir de l'eau. Les lieux sacrés où l'on campait étaient pleins de cadavres, car on mourait sur place : devant le déchaînement du mal, les hommes, ne sachant que devenir, cessèrent de rien respecter, soit de divin, soit d'humain. C'est ainsi que furent bouleversés tous les usages observés auparavant pour les sépultures : chacun ensevelissait comme il pouvait; et beaucoup eurent recours à des modes de funérailles scandaleux, car ils manquaient du nécessaire, tant il y avait déjà eu de morts autour d'eux; alors, ils profitaient de ce que d'autres avaient dressé un bûcher et, ou bien ils y plaçaient leur mort les premiers et y mettaient le feu, ou bien, tandis qu'un corps se consumait, ils jetaient dessus celui qu'ils portaient, et disparaissaient 23 .

Ce qui justifie une telle insistance et explique l'impact du texte sur les lecteurs de Thucydide jusqu'à nos jours, c'est l'importance des rites de sépulture dans toute société humaine et particulièrement dans la civilisation grecque antique; c'est aussi la volonté de Thucydide de tirer, à partir de l'histoire de ce dont il a été le témoin attentif, des enseignements durables et valables pour toute l'humanité (I, 22). Mais peut-être y a-t-il une raison supplémentaire. Ici encore, Thucydide reprend une association traditionnelle, mais d'une façon tout à fait neuve. Nous avons vu qu'une cause essentielle des pestilences, selon les oracles delphiques, réside dans des morts impies et dans le non-respect des rites de sépulture; le récit de Thucydide présente un lien de cause à effet exactement inverse : c'est la pestilence qui conduit à ne plus respecter les rites "auparavant observés" et à une situation de pollution généralisée, c'est la maladie qui "d'une façon générale, fut à l'origine ( $\pi \rho \omega \hat{\tau} \tau$ v́v $\tau \varepsilon \hat{n} \rho \xi \xi)$ d'un désordre moral croissant" $(53,1)$.

22 Voir R.C.T. PARKER, Miasma, 1983; cf. HIPPOCRATE, Mal. sacrée, I (VI, 362, 9 sq. $\mathrm{Li}=66,9$ Grensemann) par exemple, E.R. DoDDs, Les Grecs et l'Irrationnel, trad. fr., Paris, 1965 (= 1959), p. 63 n. 43; Dictionnaire étymologique de P. ChantRaine, s.v. $\mu$ í⿴囗十

23 ThuC., II, 52, 2-4 (trad. J. de RomILLY, C.U.F., 1962). 
On pourrait montrer que les observations faites par l'historien l'amènent, lorsqu'il décrit le déclenchement et le déroulement de l'épidémie, à transposer d'une certaine façon un autre thème religieux, celui de la contagion de la souillure, en une description minutieuse de la progression de la maladie par un processus de contagion entre les hommes, entre les animaux et des animaux aux hommes, qui n'a plus aucun caractère magique ${ }^{24}$. D'une façon comparable, l'historien apporte ici le témoignage qu'après vérification «dans la réalité» 25 , c'est le loimos qui entraîne la dégradation des valeurs, en particulier pour ce qui concerne la mort, et non l'inverse ${ }^{26}$.

La cité de Sybaris eut à plusieurs reprises recours à l'oracle de Delphes. Lorsqu'une pluie de sang s'abattit sur le temple d'Héra après le meurtre d'un citharède en grand costume dans le sanctuaire - cela annonçait la prise de la cité par Crotone vers 515-510 (selon une variante, Crotone prit Sybaris après le meurtre, sur les autels, de cinquante jeunes Crotoniates). Et déjà, lorsque vers 530 Crotone, Sybaris et Métaponte tuèrent cinquante jeunes de Siris réfugiés auprès de la statue d'Athéna - alors la pestilence et la guerre civile s'abattirent sur les cités 27 .

Le cas de Sybaris me permet de passer à ma troisième observation. Le loimos est souvent mis en rapport dans les oracles avec la question du salut de la cité, de la guerre civile, de la prise de la cité par un ennemi; il faut pour effacer la souillure faire des sacrifices ${ }^{28}$ de même nature que

24 Voir Notes sur... Thucydide, op. cit. n. 1, p. 342-347.

25 «De plus, ce qui auparavant se racontait dans des récits, mais se vérifiait rarement dans les faits, perdit tout caractère d'invraisemblance» (THUC., I, 23, $3:$ il s'agit des tremblements de terre, des éclipses de soleil, des sécheresses et de leurs conséquences, les famines et la pestilence athénienne). Le récit du livre II est, de façon consciente, une vérification, mais aussi une correction, d'après les faits, des traditions sur les pestilences.

26 Il est remarquable que Thucydide n'établisse, inversement, aucun lien de cause à effet entre la pestilence athénienne et la purification de Délos ainsi que le rétablissement des jeux dans l'île (THuc., III, 104).

27 Voir Fontenrose p. 76 et Q 123 (PWo 74), 126 (PWo 75). Cf. Justin, 20, 2, 3.

28 Je ne peux, faute de place, développer ce point ici et je signale seulement les sacrifices de jeunes filles exigés par l'oracle pour mettre fin à un loimos (tout comme dans la tragédie, il faut des sacrifices de jeunes filles pour sauver une armée ou une cité) : L 44, 45, 126, 134, 157 Fontenrose (PWo 209, 210, 530, 556, 332; sur le dernier cas, le fameux tribut locrien, voir l'analyse nouvelle de Fontenrose, p. 131-137); voir aussi PÉTRONE, Satiricon, I, 1. 
lorsqu'il s'agit de sauver la cité; ou encore il faut fonder une nouvelle cité pour y échapper ${ }^{29}$. Un oracle concernant Sparte montre qu'il peut y avoir substitution entre loimos et guerre civile; le dieu invite les Spartiates à faire venir Thaletas pour mettre fin, selon Plutarque, à la pestilence, selon Philodème, à la guerre civile ${ }^{30}$. Un autre oracle associe comme pour Sybaris loimos et stasis : selon une tradition, le roi Iphitos d'Élis consulta pour savoir comment mettre fin à la guerre civile et à la pestilence qui ravageaient la Grèce : il dut rétablir les Jeux Olympiques ${ }^{31}$. Ce lien est très fortement senti dès Eschyle au moins, comme en témoignent un et peut-être deux emplois du mot loimos chez lui : dans les Perses, Darius ne voit que deux calamités qui puissent avoir anéanti la puissance perse, le loimos ou la stasis (v. 715); dans le second stasimon des Suppliantes, le parallélisme entre les seconde et troisième strophes suggère que le loimos mentionné au v. 659 est ici aussi associé à l'évocation de la guerre civile (au v. 661) ${ }^{32}$.

$\mathrm{Si}$, à nouveau, nous revenons à Thucydide, nous observons d'abord qu'il associe lui aussi stasis et loimos dans le chapitre 23 du livre I où il affirme le caractère exceptionnel de la guerre du Péloponnèse : ce chapitre présente en effet deux grands mouvements, dont le premier concerne la dévastation des cités $(\$ 1-2)$ et le second les grands cataclysmes naturels $(\$ 3)$ : le premier mouvement se termine par la mention de la guerre civile, le second par celle de la pestilence. D'autre part, le récit de la guerre du Péloponnèse présente à propos de la pestilence et à propos des guerres civiles deux analyses comparables, et

29 Cela montre bien que le loimos est conçu comme une sorte de négation de la cité; voir Q 94 et L 72 Fontenrose (PWo $401-402$ et 305; cf. FGrH 327 F 17-). Par ailleurs, cela pouvait s'accorder avec les analyses médicales de la pestilence comme infection de l'air (il faut alors changer d'air), analyses qui reprennent sur un mode rationnel nouveau le thème religieux de la souillure, et avec le même vocabulaire (cf. par ex. le traité hippocratique Vents, VI, p. 109110 Jouanna, avec les notes).

30 Voir Plut., Mor., 1146c; PhILod., Mus., 4. 18, 37-38 (Fontenrose Q 54; PWo 223).

31 Cf. Q 1 Fontenrose (PWo 485); cf. PAUs., V, 4, 6. Dans une autre version, c'est Lycurgue qui présente la demande, à propos d'une pestilence et d'une famine, avec la même réponse (Q 3 Fontenrose, PWo 487).

32 C'est pourquoi je choisis, avec G. MURRAY, l'adjonction de BAMBERGER (stasis) ou toute autre de même nature (eris HEATH, WIL.; WILAMOWITZ condamne la correction de BAMBERGER, qui ferait double emploi avec le v. 680; mais il y a un parallélisme très net; cf. TUCKER ad locum). Un curieux exemple de l'association entre loimos et stasis (et d'ailleurs aussi limos et loimos), que m'a signalé V. Fromentin, se trouve dans DenYs D'HALICARNASSE (I, 22-24). 
souvent comparées, de la dégradation des valeurs qu'elles entraînent l'une et l'autre (II, 53 et III, 82 sqq.). Il est inutile, et impossible d'ailleurs dans le cadre de cette communication, de reprendre ici ces deux textes fameux. Constatons seulement que les observations de Thucydide s'inscrivent, comme dans les cas précédents, à l'intérieur d'une conception du monde où pestilence et guerre civile sont traditionnellement associées. Ici, la rationalisation consiste encore pour une part à renverser l'ordre des causes : ces deux fléaux ne sont pas le châtiment de fautes qu'on doit expier, mais les causes de la destruction des valeurs morales et religieuses.

Lire le récit de Thucydide après les traditions concernant les oracles delphiques ne renouvelle certes pas l'interprétation de l'historien. Cela permet cependant peut-être, c'était en tout cas le but de cette communication, de progresser dans la compréhension de la façon dont, à propos d'un événement si chargé de sens magique et religieux pour ses contemporains, Thucydide a mis en œuvre ses remarquables facultés d'observation et d'analyse rationnelles en son temps, à partir des différentes conceptions du monde en vigueur autour de lui, y compris les plus traditionnelles. Observons d'ailleurs qu'il cite à trois reprises un oracle d'Apollon aux Lacédémoniens annonçant que le dieu les aiderait personnellement à faire la guerre aux Athéniens ${ }^{33}$. À la première mention ( $\mathrm{I}, 118,3$ ), il y a peut-être une réserve : «à ce qu'on dit»; mais le roi Archidamos y fait allusion dans son discours au Congrès de Sparte (I, 123, 1), ce qui implique que pour Thucydide cet oracle a bien existé dès avant le déclenchement des hostilités. Aussi, lorsque des "gens au courant» s'en souviennent à Athènes au moment de la pestilence, trouve-t-il "naturel" qu'on y pense (mais cette fois sans imaginer une autre interprétation possible qui serait tout aussi naturelle dans d'autres circonstances) :

On évoqua aussi, chez les gens au courant, l'oracle rendu aux Lacédémoniens, quand ils avaient demandé au dieu s'ils devaient faire la guerre : il avait répondu que s'ils la faisaient avec énergie, ils auraient la victoire ${ }^{34}$; et il avait promis de prêter lui-même son

33 On notera aussi que, lorsque THUCYDIDE signale la réapparition de la pestilence à III, 87, il y joint la mention de divers tremblements de terre, d'une façon assez comparable à ce qu'il fait dans le prologue à I, 23.

34 Le dieu voyait juste. Mais ceci est une autre histoire. Sur la façon dont les Spartiates durent renoncer à la tranquillité pour battre Athènes, voir 
appui. En ce qui concerne l'oracle, on trouvait donc dans les événements une confirmation; en fait, l'épidémie avait commencé sitôt l'invasion péloponnésienne en cours; elle ne gagna pas le Péloponnèse de façon qui mérite d'être mentionnée 35 ; elle envahit principalement Athènes, et après elle, les parties les plus peuplées des autres régions (II, 54, 4-5).

Université d'Amiens

Paul DEMONT

Faculté des Lettres

Campus Universitaire

F - 80025 AMIENs Cédex

P. DEMONT, La cité grecque archaïque et classique et l'idéal de tranquillité (à paraitre courant 1989, Paris, Les Belles-Lettres).

35 La sécheresse du constat ne doit pas conduire à sous-estimer cette remarque. La sélectivité de l'épidémie interdit en fait d'exclure une explication surnaturelle. On a pu observer, dans un tout autre contexte, «l'effet paralysant» des premieres épidémies de variole introduites en Amérique par les envahisseurs espagnols, épidémies «qui tuaient les Indiens sans affecter les Espagnols» (W.H. MACNEILL, op. cit., p. 8 et 183-185). Mais Athènes, malgré la violence du choc (cf. THUC., III, 87), tint bon, à la stupéfaction générale. 